\title{
ON INJECTIVITY IN LOCALLY PRESENTABLE CATEGORIES
}

\author{
JIŘí ADÁMEK AND JIŘí ROSICKÝ
}

\begin{abstract}
Classes of objects injective w.r.t. specified morphisms are known to be closed under products and retracts. We prove the converse: a class of objects in a locally presentable category is an injectivity class iff it is closed under products and retracts. This result requires a certain large-cardinal principle.

We characterize classes of objects injective w.r.t. a small collection of morphisms: they are precisely the accessible subcategories closed under products and $\kappa$-filtered colimits. Assuming the (large-cardinal) Vopènka's principle, the accessibility can be left out. As a corollary, we solve a problem of L. Fuchs concerning injectivity classes of abelian groups. Finally, we introduce a weak concept of reflectivity, called cone reflectivity, and we prove that under Vopěnka's principle all subcategories of locally presentable categories are cone reflective.

Several open questions are formulated, e.g., does each topological space have a largest (non- $T_{2}$ ) compactification?
\end{abstract}

\section{INTRODUCTION}

0.1. Recall that an object $X$ is said to be injective w.r.t. of class $\mathscr{M}$ of morphisms provided that for each $m: A \rightarrow A^{\prime}$ in $\mathscr{M}$ and each morphism $f: A \rightarrow X$ there exists a (not necessarily unique) morphism $f^{\prime}: A^{\prime} \rightarrow X$ with $f=f^{\prime} \cdot m$. The collection of all objects injective w.r.t. a given class of morphism is called an injectivity class. This concept has been introduced by J.-M. Maranda [M] who proved, inter alia, that every injectivity class is closed under products and retracts. The converse is false, in general. For example in the category of topological spaces the class of all connected spaces is closed under products and retracts, although it is not an injectivity class. We will prove that no such (constructive) example can be found in the realm of locally presentable categories.

Another obvious fact is that in any category $\mathscr{K}$ each reflective subcategory ${ }^{1}$ is an injectivity class: put $\mathscr{M}=$ all reflection arrows. This generalizes to weakly reflective subcategories, i.e., subcategories $\mathscr{A}$ of $\mathscr{K}$ closed under retracts and such that each object $K$ in $\mathscr{K}$ has a weak reflection $r: K \rightarrow K^{*}$ (i.e., $K^{*} \in A$ and every morphism $f: K \rightarrow A, A \in \mathscr{A}$, factorizes through $r$, not necessarily uniquely). Every weakly reflective subcategory is an injectivity class. The converse is false, in general. For example in the category of frames the class of all complete Boolean algebras is an injectivity class which is not weakly reflective.

Received by the editors April 2, 1990 and, in revised form, December 28, 1990.

1980 Mathematics Subject Classification (1985 Revision). Primary 18A40, 18A99, 08C05, 03 E55. The hospitality of the Université Catholique de Louvain-la-Neuve is gratefully acknowledged.

${ }^{1}$ All subcategories are understood to be full and isomorphism closed throughout the paper. 
We will prove that no such (constructive) example can be found in the realm of locally presentable categories.

0.2. Recall that Vopernka's principle is the following statement: the category Gra of graphs (= sets with a binary relation) does not have a large discrete subcategory. This is a large cardinal principle: the existence of huge cardinals implies that Vopěnka's principle is consistent, and Vopěnka's principle implies the existence of arbitrarily large measurable (or even compact) cardinals, see [J]. The above remarks about the absence of constructive examples in locally presentable categories $\mathscr{K}$ can be made precise as follows: Assuming Vopěnka's principle, every class of objects of $\mathscr{K}$ closed under products and retracts is an injectivity class, in fact, is weakly reflective. This answers a problem put by $\mathrm{L}$. Fuchs about injectivity in the category of abelian groups.

Recall further weak Vopernka's principle: it states that Ord $^{\text {op }}$ (the dual to the usual well-ordered class of all ordinals) cannot be fully embedded into Gra . We have proved in [ART] that the weak Vopenka's principle implies (in fact, is equivalent to) the following statement on locally presentable categories $\mathscr{K}$ : every class of objects closed under small limits forms a reflective subcategory. The exact set-theoretical position of this principle is an open problem, but it has been shown in [ART] that Vopěnka's principle implies weak Vopěnka's principle which, in turn, implies the existence of arbitrarily large measurable cardinals. (Whether it implies compact cardinals is not known.) The above statement concerning injectivity classes turns out to "lie between" the two principles. That is, assuming the negation of weak Vopěnka's principle, there exists an injectivity class in a locally presentable category, which is not weakly reflective.

We introduce below another variant, called semiweak Vopěnka's principle, which exactly describes the set-theoretical status of the above statement on injectivity classes.

0.3. A concept very near to injectivity is that of orthogonality. Recall that an object $X$ is said to be orthogonal w.r.t. a class $\mathscr{M}$ of morphisms provided that for each $m: A \rightarrow A^{\prime}$ in $\mathscr{M}$ and each morphism $f: A \rightarrow X$ there exists a unique morphism $f^{\prime}: A^{\prime} \rightarrow X$ with $f=f^{\prime} \cdot m$. The collection of all objects orthogonal w.r.t. a given class of morphisms is called an orthogonality class. In each category $\mathscr{K}$ one has the following implications for properties of subcategories:

$$
\begin{aligned}
& \text { reflective } \quad \Rightarrow \text { orthogonality class } \Rightarrow \text { closed under limits } \\
& \Downarrow \quad(*) \Downarrow \quad \Downarrow \\
& \text { weakly reflective } \Rightarrow \text { injectivity class } \Rightarrow \text { closed under products } \\
& \text { and retracts }
\end{aligned}
$$

(They hold trivially and generally except $(*)$ which, as will be seen below, requires that $\mathscr{K}$ have pushouts.) Both of the implications in the first row can be reversed if $\mathscr{K}$ is locally presentable and if the weak Vopenka's principle is assumed, see [ART]). (Again, the weak Vopěnka's principle is actually equivalent to the possibility of reverting any one, or both, of those implications, see [AR 3 .)

0.4. Interesting concepts arise when we restrict the morphism classes $\mathscr{M}$ under consideration to small ones. A class of all objects injective (or orthogonal) w.r.t. a given small collection of morphisms is called a small-injectivity class 
(or a small-orthogonality class, respectively). In a locally presentable category $\mathscr{K}$ every small-injectivity class, and every small-orthogonality class, is easily seen to be accessibly embedded, i.e., closed under $\kappa$-filtered colimits for some regular cardinal $\kappa$. We have proved in $\left[\mathrm{AR}_{2}\right]$ that in the orthogonality case this actually characterizes small orthogonality (independently of set theory). If $\mathscr{K}$ is a locally presentable category, then small-orthogonality classes in $\mathscr{K}$ are precisely the accessibly embedded classes closed under limits. It then follows from [RTA] that, assuming Vopěnka's principle, each reflective subcategory is a small-orthogonality class. Surprisingly, analogous results are not true for smallinjectivity. For example, the injectives in the category of posets are precisely the complete lattices, and this is not a small-injectivity class. Furthermore, assuming the negation of the weak Vopenka's principle, we present a class of objects of Gra which is accessibly embedded and closed under products and retracts, and yet, it is not a small-injectivity class.

The absolute results we prove about small-injectivity classes are the following: in the realm of locally ranked categories (which is a broad collection of categories introduced below and includes, besides locally presentable categories, for example Top) every small-injectivity class is weakly reflective. And in the realm of locally presentable categories, small-injectivity classes are precisely the accessibly embedded classes closed under products which, moreover, are accessible in the sense of M. Makkai and R. Paré [MP], see also [L]. Accessibility can be left out assuming Vopěnka's principle.

0.5. We call a subcategory of a category $\mathscr{K}$ cone reflective provided that the inclusion functor into $\mathscr{K}$ satisfies the solution-set condition. It turns out that, assuming Vopěnka's principle, every subcategory of a locally presentable category is cone reflective.

\section{INJECTIVITY CLASSES AND WEAK REFLECTIVITY}

I.1 Definition. For each class $\mathscr{M}$ of morphisms of a category $\mathscr{K}$ let $\mathscr{M}$-Inj denote the collection of all $\mathscr{K}$-objects $K$ which are $\mathscr{M}$-injective, i.e., such that for each $m: A \rightarrow A^{\prime}$ in $\mathscr{M}$ and each morphism $f: A \rightarrow K$ there exists a morphism $f^{\prime}: A^{\prime} \rightarrow K$ with $f=f^{\prime} \cdot m$. An injectivity class is a class of objects of $\mathscr{K}$ of the form $\mathscr{M}$-Inj for some collection $\mathscr{M}$ of morphisms of $\mathscr{K}$. For $\mathscr{M}=$ all monomorphisms, $\mathscr{M}$-injective objects are called injective.

I.2 Examples. (1) Injective objects in the category $\mathrm{Ab}$ of abelian groups are the divisible groups. (Pure embedding)-injective objects are the algebraically compact groups, see [Fu].

(2) [BB] Complete lattices form an injectivity class in Pos, the category of posets and order-preserving maps. Here $\mathscr{M}=$ Strong Mono. (In contrast, Mono-Inj is just the class of all singleton posets.)

(3) Complete Boolean algebras form an injectivity class in Frm, the category of frames and frame homomorphisms (cf. [Jo]): here $\mathscr{M}$ consists of the single embedding pictured in Figure 1.

(4) If $\mathscr{K}$ is a complete, cowellpowered category, then epireflective subcategories (i.e., $S P$ classes in $\mathscr{K}$ ) are precisely the injectivity classes $\mathscr{M}$-Inj where $\mathscr{M} \subseteq$ Epi.

We will later see that every reflective subcategory of a category $\mathscr{K}$ is an injectivity class, whenever $\mathscr{K}$ has pushouts. 


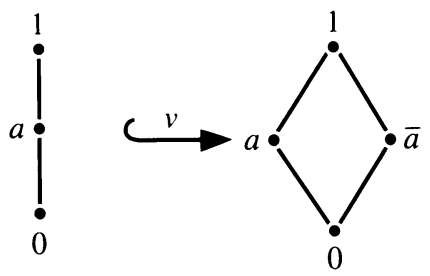

FIGURE 1

(5) [H. Herrlich]. Pathwise connected spaces form an injectivity class in Top: here $\mathscr{M}$ is the single embedding $\{0,1\} \rightarrow[0,1]$ where $[0,1 \mid$ is the usual interval of the real line.

In contrast, all connected spaces do not form an injectivity class in Top . (Proof. Let $m: A \rightarrow A^{\prime}$ be a continuous map such that every connected space is $\{m\}$-injective. Using sufficiently large connected spaces with all subspaces of cardinality card $A^{\prime}$ discrete, it is easy to show that for each clopen $U \subseteq A$ there is a clopen $U^{\prime} \subseteq A^{\prime}$ with $U=m^{-1}\left(U^{\prime}\right)$. It follows that the discrete two-point space is also $\{m\}$-injective.)

I.3 Proposition [M]. Each injectivity class is closed under the formation of products and retracts.

Proof. Let $I$ be a set. Given $X_{i} \in \mathscr{M}$-Inj $(i \in I)$, then $X=\prod_{i \in I} X_{i} \in \mathscr{M}$-Inj . For each $m: A \rightarrow A^{\prime}$ in $\mathscr{M}$, each $f: A \rightarrow X$ and each $i \in I$ we have $f_{i}^{\prime}: A^{\prime} \rightarrow$ $X_{i}$ with $\pi_{i} \cdot f=f_{i}^{\prime} \cdot m$ (where $\pi_{i}$ denotes the projections), and the morphism $f^{\prime}: A^{\prime} \rightarrow \prod_{i \in I} X_{i}$ with components $f_{i}^{\prime}$ fulfils $f=f^{\prime} \cdot m$.

Give $X \in \mathscr{M}$-Inj and a retraction $r: X \rightarrow Y$ (with $j: Y \rightarrow X$ satisfying $r \cdot j=$ id), then $Y \in \mathscr{M}$-Inj. For each $m: A \rightarrow A^{\prime}$ in $\mathscr{M}$ each $f: A \rightarrow Y$ we have $f^{\prime}: A^{\prime} \rightarrow X$ with $j \cdot f=f^{\prime} \cdot m$, and then $r \cdot f^{\prime}: A^{\prime} \rightarrow Y$ fulfils $f=\left(r \cdot f^{\prime}\right) \cdot m$.

I.4 Example. Connected spaces are well known to be closed in Top under products and retracts, although we have seen in I. 2 that they are not an injectivity class. Such a (constructive) example cannot be found in a locally presentable category $\mathscr{K}$, as we prove in I.9.

I.5 Definition. A subcategory $\mathscr{A}$ of $\mathscr{K}$ is called weakly reflective if for each object $K$ of $\mathscr{K}$ there exists a morphism $r: K \rightarrow K^{*}$ such that

(i) $K^{*} \in \mathscr{A}$ and for each morphism $f: K \rightarrow A, A \in \mathscr{A}$, there exists a (not necessarily unique) morphism $f^{*}: K^{*} \rightarrow A$ with $f=f^{*} \cdot r$,

(ii) if $r$ is a split mono, then $K \in \mathscr{A}$.

I.6 Remark. (a) Condition (ii) in the above definition means that the subcategory is closed under retracts. A morphism satisfying (i) is called a weak reflection of $\mathscr{K}$.

(b) Recall that, for a class $\mathscr{M}$ of morphisms in $\mathscr{K}$, an $\mathscr{M}$-injective hull of an object $K \in \mathscr{K}$ is an $\mathscr{M}$-essential morphism $m: K \rightarrow \widehat{K}$ with $\widehat{K} \mathscr{M}$-injective (where a morphism $m: K \rightarrow L$ is called $\mathscr{M}$-essential provided that for any morphism $f: L \rightarrow L^{\prime}$ in $\mathscr{K}$ such that $f \cdot m \in \mathscr{M}$ it follows that $\left.f \in \mathscr{M}\right)$. A class of objects may be called an "injectivity class with injective hulls" provided 
that it has the form $\mathscr{M}$-Inj for some collection $\mathscr{M}$ of morphisms such that each $\mathscr{K}$-object has an $\mathscr{M}$-injective hull. This is tantamount to the weak reflectivity of the corresponding full subcategory.

Indeed, if $\mathscr{A}$ is weakly reflective, choose a weak reflection $m_{K}: K \rightarrow \widehat{K}$ for each $\mathscr{K}$-object $K$, and put $\mathscr{M}=\left\{m_{K}\right\}_{K \in \mathscr{K}}$. Conversely, if $A=\mathscr{M}$-Inj and each $\mathscr{K}$-object has an injective hull, then that hull is a weak reflection, and any retract of an $\mathscr{M}$-injective object is $\mathscr{M}$-injective.

(c) Let $\mathscr{A}$ be a weakly reflective subcategory of $\mathscr{K}$. Then $\mathscr{A}$ is, obviously, closed under products. Thus, $\mathscr{A}$ is reflective iff it is closed under equalizers. (In fact, if $\mathscr{A}$ is closed under equalizers, it is closed under limits. Given a weak reflection $r: K \rightarrow A$ of an object $K$, let $e: A_{0} \rightarrow A$ be the joint equalizer of all endomorphisms $a$ of $A$ with $r=r \cdot a$. The unique $r_{0}: K \rightarrow A_{0}$ with $r=e \cdot r_{0}$ is a reflection of $K$.)

I.7 Examples. (i) In the category of modules the injective modules are weakly reflective: injective hulls are weak reflections. Analogously, the Mac Neille completion defines a weak reflection of a poset in the category of complete lattices.

(ii) Complete Boolean algebras are not weakly reflective in Frm (because, by I.6(c), they would be reflective, but that would imply the existence of free complete Boolean algebras.) Yet, they form an injectivity class. Such a (constructive) example cannot be found in a locally presentable category, as we prove in I.9.

I.8 Remark. Vopěnka's principle (see introduction) has a number of equivalent formulations. For example, in model theory it is formulated as follows: Every class of models of a first-order theory with no nontrivial elementary embeddings is a set. Here are some other formulations (see [ART]):

(i) No locally presentable category has a large discrete subcategory;

(ii) Ord cannot be fully embedded into Gra;

(iii) Ord cannot be fully embedded into any locally presentable category.

I.9 Theorem. Assuming Vopěnka's principle, for each subcategory $\mathscr{A}$ of a locally presentable category, the following are equivalent.

(i) $\mathscr{A}$ is an injectivity class.

(ii) $\mathscr{A}$ is weakly reflective.

(iii) $\mathscr{A}$ is closed under products and retracts.

Proof. The implications (ii) $\rightarrow($ i) $\rightarrow$ (iii) are obvious. To prove (iii) $\rightarrow$ (ii), let $\mathscr{K}$ be locally presentable and let $\mathscr{A}=\left\{A_{i}\right\}_{i \in \text { Ord }}$ be a collection of objects closed under products and retracts. For each ordinal $i$, let $\mathscr{B}_{i}$ be the collection all retracts of products of the objects $A_{j}(j \leq i)$. Then $\mathscr{B}_{i}$ is weakly reflective in $\mathscr{K}$ : for each object $K$ in $\mathscr{K}$ the canonical morphism

$$
K \rightarrow \prod_{j \leq i} A_{j}^{\operatorname{hom}\left(K, A_{j}\right)}
$$

is, obviously, a weak reflection, and $\mathscr{B}_{i}$ is closed under retracts.

Suppose that $\mathscr{A}$ is not weakly reflective. Since $\mathscr{A}$ is closed under retracts, this means that there is an object $K$ in $\mathscr{K}$ which does not have a weak reflection in $\mathscr{A}$. However, $K$ has weak reflections $r_{i}: K \rightarrow K_{i}^{*}$ in $\mathscr{B}_{i}$ for each $i$. 
Consequently, for each ordinal $i$ there exists an ordinal $d(i)>i$ such that $r_{d(i)}$ does not factor through $r_{i}$ (since otherwise $r_{i}: K \rightarrow K_{i}^{*}$ would be a weak reflection in each $\mathscr{B}_{j}$, and hence, in $\mathscr{A}$.) On the other hand, observe that since $j \leq i$ implies $\mathscr{B}_{j} \subseteq \mathscr{B}_{i}$, by the definition of weak reflection $r_{j}$ factors through $r_{i}$. Consequently, in the comma category $K \downarrow \mathscr{K}$ (of all arrows with the domain $K$ ) we can define objects $L_{i}, i \in$ Ord, as follows:

$$
L_{0}=K \stackrel{r_{0}}{\longrightarrow} K_{0}^{*}, \quad L_{i}=K \stackrel{r_{d(j)}}{\longrightarrow} K_{d(j)}^{*},
$$

where $j$ is the join of all ordinals $k$ such that for some $i^{\prime}<i, L_{i^{\prime}}=K \stackrel{r_{k}}{\rightarrow} K_{k}^{*}$. Then $\operatorname{hom}\left(L_{i}, L_{j}\right) \neq \varnothing$ whenever $j \leq i$ by the above factorization of weak reflections, but $\operatorname{hom}\left(L_{i}, L_{j}\right)=\varnothing$ whenever $j>i$ (because of the choice of $d(-))$. Briefly, in $K \downarrow \mathscr{K}$ we have

$$
\operatorname{hom}\left(L_{i}, L_{j}\right) \neq \varnothing \quad \text { iff } \quad i \geq j .
$$

Since $\mathscr{K}$ is locally presentable, so is $K \downarrow \mathscr{K}$, and hence, by [ART], $K \downarrow \mathscr{K}$ can be fully embedded into Gra. By $(*)$, we have graphs $\left(X_{i}, \alpha_{i}\right), i \in$ Ord, such that for $i<j$, card $X_{i}<\operatorname{card} X_{j}$ and there is no morphism $\left(X_{i}, \alpha_{i}\right) \rightarrow$ $\left(X_{j}, \alpha_{j}\right)$. In the category $\operatorname{Rel}(2,2,2)$ of three binary relations we have a large discrete subcategory consisting of objects $\left(X_{i}, \alpha_{i}, \beta_{i}, \gamma_{i}\right)$ where $\beta_{i}$ is rigid and $\gamma_{i}$ is the relation $\neq$. This contradicts to Vopěnka's principle.

I.10 Remark. The full strength of Vopěnka's principle has not been used in the proof of I.9. The following (possibly weaker) principle is sufficient.

I.11 Definition. The semiweak Vopernka's principle is the following statement: There do not exist graphs $L_{i} \quad(i \in$ Ord) such that in Gra,

$$
\operatorname{hom}\left(L_{i}, L_{j}\right) \neq \varnothing \quad \text { iff } \quad i \geq j .
$$

I.12 Observation. The three Vopenka's principle (VP) are related by

$$
\mathrm{VP} \Rightarrow \text { semiweak VP } \Rightarrow \text { weak VP. }
$$

In fact, the first implication follows from the proof of I.9, and the latter is clear.

In I.9 we have proved that the semiweak Vopěnka's principle implies "injectivity class = weakly reflective subcategory". We will now show that the statement is actually equivalent to the semiweak Vopěnka's principle.

I.13 Theorem. Assuming the negation of the semiweak Vopernka's principle, there exists an injectivity class in a locally finitely presentable category which is not weakly reflective.

Proof. The negation of the semiweak Vopěnka's principle provides us

(i) with graphs $L_{i} \quad\left(i \in\right.$ Ord) such that $\operatorname{hom}\left(L_{i}, L_{j}\right) \neq \varnothing$ iff $i \geq j$ and

(ii) with a large discrete category of graphs $K_{i} \quad(i \in$ Ord) (since it implies the negation of Vopěnka's principle, see I.11). Put $K_{i}=\left(X_{i}, \alpha_{i}\right)$ and $L_{i}=\left(Y_{i}, \beta_{i}\right) \quad(i \in$ Ord $)$.

In the category $\operatorname{Str}(2,2,1)$ of structures with two binary and one unary relation define objects $A_{i} \quad(i \in$ Ord $)$ as follows.

$$
A_{i}=\left(X_{i}+Y_{i}+\left\{t_{i}\right\}, \alpha_{i} \cup Y_{i} \times\left\{t_{i}\right\}, \beta_{i} \cup X_{i} \times\left\{t_{i}\right\},\left\{t_{i}\right\}\right),
$$


where, for simplicity of notation, we assume $X_{i} \cap Y_{i}=\varnothing$ and $t_{i} \notin X_{i} \cup Y_{i}$, thus, the underlying set of $A_{i}$ is $X_{i} \cup Y_{i} \cup\left\{t_{i}\right\}$. For each ordinal $i$ put $\bar{A}_{i}=$ $\amalg_{j \leq i} A_{i}$, and let $v_{i}: A_{0} \rightarrow \bar{A}_{i}$ be the coproduct injection. We will prove that for $\mathscr{M}=\left\{v_{i} \mid i \in\right.$ Ord $\}, \mathscr{M}$-Inj is not weakly reflective. We first define objects $B_{i}$ which lie in the injectivity class. In $\bar{A}_{i}$ merge all points of $X_{i}$ to one (denoted $\left.s_{i}\right)$ :

$$
B_{i}=\coprod_{j<i} A_{j}+\left(\left\{s_{i}\right\}+Y_{i}+\left\{t_{i}\right\},\left\{\left(s_{i}, s_{i}\right)\right\} \cup Y_{i} \times\left\{t_{i}\right\}, \beta_{i} \cup\left\{\left(s_{i}, t_{i}\right)\right\},\left\{t_{i}\right\}\right) .
$$

Observe that $\operatorname{hom}\left(A_{j}, B_{i}\right) \neq \varnothing$ for all $i, j \in$ Ord: For $j<i$ we have a coproduct injection, for $j \geq i$ there is $f: L_{j} \rightarrow L_{i}$, and we define $\bar{f}: A_{j} \rightarrow B_{i}$ by $\bar{f}(x)=s_{i}$ for $x \in X_{j}, \bar{f}(y)=f(y)$ for $y \in Y_{j}$ and $\bar{f}\left(t_{j}\right)=t_{i}$. It follows easily that $B_{i} \in \mathscr{M}$-Inj. Observe also that

(*) $j<i$ implies that for each morphism $f: A_{j} \rightarrow B_{i}$ there exists a morphism $g: L_{j} \rightarrow L_{j}$ such that $f(x)=x$ for $x \in X_{j} \cup\left\{t_{j}\right\}$ and $f(y)=g(y)$ for $y \in Y_{j}$.

In fact, due to the unary relation of $A_{j}, f\left(t_{j}\right)=t_{j^{\prime}}$ for some $j^{\prime} \leq i$. Since the first binary relation contains $Y_{j} \times\left\{t_{j}\right\}$, it follows that $f\left(Y_{j}\right) \subseteq Y_{j^{\prime}}$, and then the restriction of $f$ defines a morphism $L_{j} \rightarrow L_{j^{\prime}}$. Consequently, $j \geq j^{\prime}$ (thus, $\left.j^{\prime} \neq i\right)$. Analogously, since the second binary relation contains $X_{j} \times\left\{t_{j}\right\}$, we have $f\left(X_{j}\right) \subseteq X_{j^{\prime}}$, and the restriction of $f$ defines a morphism $K_{j} \rightarrow K_{j^{\prime}}$. Consequently, $j=j^{\prime}$ and $f(x)=x$ for $x \in X_{j} \cup\left\{t_{j}\right\}$.

We now prove that $A_{0}$ does not have a weak reflection in $\mathscr{M}$-Inj. Let, to the contrary, $r: A_{0} \rightarrow A_{0}^{*}$ be a weak reflection. Since $A_{0}^{*} \in \mathscr{M}$-Inj, for each ordinal $j$ there exists a morphism $p_{j}: A_{j} \rightarrow A_{0}^{*}$. We will show that $j \neq j^{\prime}$ implies $p_{j}\left(t_{j}\right) \neq p_{j^{\prime}}\left(t_{j^{\prime}}\right)$, which is clearly impossible. Choose an ordinal $i$ larger than both $j$ and $j^{\prime}$. For the morphism $f: A_{0} \rightarrow B_{i}$ which is the coproduct injection there exists, by the definition of weak reflection, $f^{*}: A_{0}^{*} \rightarrow B_{i^{\prime}}$ and from $(*)$ above we know that $f^{*} \cdot p_{j}\left(t_{j}\right)=t_{j}$ and $f^{*} \cdot p_{j^{\prime}}\left(t_{j^{\prime}}\right)=t_{j^{\prime}}$. Thus, $p_{j}\left(t_{j}\right) \neq p_{j^{\prime}}\left(t_{j^{\prime}}\right)$.

I.14 Remark. (a) The proof above makes use of a technique applied in our paper [ART].

(b) We do not know the set-theoretical status of the statement "injectivity class = class closed under products and retracts".

In contrast, for reflective subcategories of locally presentable categories we have proved in [ART and $\mathrm{AR}_{3}$ ] that

(i) Weak Vopěnka's principle implies that "reflective $=$ orthogonal $=$ closed under limits";

(ii) Each of the two equality statements implies the weak Vopernka's principle.

I.15 Corollary. The semiweak Vopernka's principle is equivalent to the following statement: every class of objects of a locally presentable category, closed under products and retracts, is weakly reflective.

Now we turn to the injectivity w.r.t. monomorphisms. Recall that injective means $\mathscr{M}$-injective for $\mathscr{M}=$ all monomorphisms.

I.16 Definition. A category is said to have enough injectives provided that every object is a subobject of some injective object. 
I.17 Corollary. Let the semiweak Vopernka's principle hold. Then in each locally presentable category with enough injectives the following conditions on a class $\mathscr{A}$ of objects is equivalent:

(i) $A=\mathscr{M}$-Inj for some collection $\mathscr{M}$ of monomorphisms,

(ii) $\mathscr{A}$ is closed under products and retracts and contains all injectives.

In fact, (i) $\rightarrow$ (ii) is clear. If (ii) holds, then $\mathscr{A}$ is weakly reflective and each object is a subobject of an $\mathscr{A}$-object. Thus, weak reflections are monomorphisms, and $\mathscr{A}=\mathscr{M}$-Inj for $\mathscr{M}=$ all weak reflections.

I.18 Example. Assuming the semiweak Vopěnka's principle, injectivity classes of abelian groups with respect to collections of embeddings are precisely the classes closed under products and retracts, and containing all divisible groups. This answers Problem 46 of L. Fuchs [Fu].

I.19 Remark. In the Introduction we mentioned the observation of $\mathrm{H}$. Herrlich that connected topological spaces do not form an injectivity class of Top although they are closed in Top under products and retracts.

We do not know any example of an injectivity class in Top which is not weakly reflective.

In fact, the following, much more concrete (and rather fascinating) question is open. Is the class of all compact spaces weakly reflective? And still more concretely. Does the discrete countable space have a weak compact reflection? (By another observation of $\mathrm{H}$. Herrlich, compact spaces do form an injectivity class: let $\mathscr{M}$ be the collection of all embeddings $X \rightarrow X \cup\{a\}$ where $X$ is discrete and the neighborhoods of $a$ form an ultrafilter on $X$.)

In contrast, an example of an orthogonality class in Top which is not reflective has been presented in $\left[\mathrm{AR}_{1}\right]$.

\section{SMALl-orthogonality CLASSES}

II.1 Definition. Given a class $\mathscr{M}$ of morphisms, $\mathscr{M}^{\perp}$ denotes the class of objects orthogonal to each $m: A \rightarrow A^{\prime}$ in $\mathscr{M}$ (i.e., for every $f: A \rightarrow X$ there exists a unique $f^{\prime}: A^{\prime} \rightarrow X$ with $\left.f=f^{\prime} \cdot m\right)$. Classes of the form $\mathscr{M}^{\mathrm{L}}$ are called orthogonality classes, classes of the form $\mathscr{M}^{\perp}$ where $\mathscr{M}$ is small are called small-orthogonality classes.

Analogously, classes of the form $\mathscr{M}$-Inj where $\mathscr{M}$ is small are called smallinjectivity classes.

II.2 Examples. (1) Every reflective subcategory is an orthogonality class. Set $\mathscr{M}=$ all reflection arrows. In Top there exist nonreflective orthogonality classes, see $\left[\mathrm{AR}_{1}\right]$.

(2) Denote by $m$ the empty map from the empty algebra to a singleton algebra, say, in $\operatorname{Alg}(1)$ (the category of unary algebras on one operation). Then $\{m\}^{\perp}$ is the class of all algebras with a single fixpoint; whereas $\{m\}$-Inj is the class of all algebras with at least one fixpoint. Observe that $\{m\}^{\perp}$ is reflective but $\{m\}$-Inj is only weakly reflective. Further observe that $\{m\}^{\perp}$ is an injectivity class. If $n$ denotes the quotient map of the algebra $(\{0,1\}$, id) to the singleton algebra, then $\{m\}^{\perp}=\{m, n\}$-Inj .

This is no coincidence: 
II.3 Proposition. In a category with pushouts every (small) orthogonality class is a (small) injectivity class.

Proof. Given a morphism $m: A \rightarrow A^{\prime}$, let us form the pushout of $m$ with itself

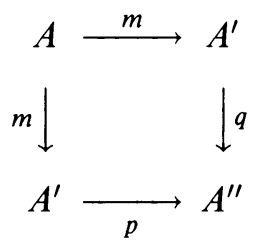

Let $\hat{m}: A^{\prime \prime} \rightarrow A^{\prime}$ be the unique morphism with $\hat{m} \cdot p=\hat{m} \cdot q=\mathrm{id}_{A^{\prime}}$. It is obvious that $\{m\}^{\perp}=\{m, \hat{m}\}$-Inj .

Given a collection $\mathscr{M}$ of morphisms, then $\mathscr{M}^{\perp}=(\mathscr{M} \cup\{\hat{m} \mid m \in \mathscr{M}\})$-Inj .

II.4 Remark. (a) The hypothesis in II.3 that $\mathscr{K}$ have pushouts is essential. In the following category

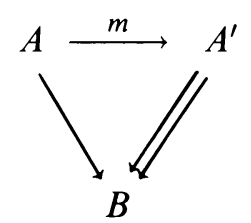

$\{m\}^{\perp}=\left\{A^{\prime}\right\}$ is not an injectivity class.

(b) In a category such that $\operatorname{hom}(A, B) \neq \varnothing$ for arbitrary objects $A, B$ "small" can be reduced to "singleton": if $\mathscr{M}$ is a set of morphisms $m_{i}: A_{i} \rightarrow$ $B_{i}$, then for $m=\coprod m_{i}: \coprod A_{i} \rightarrow \coprod B_{i}$, we have $\mathscr{M}$-Inj $=\{m\}$-Inj and $\mathscr{M}^{\perp}=$ $\{m\}^{\perp}$.

A subcategory $\mathscr{A}$ is called accessibly embedded in a category $\mathscr{K}$ it if is closed under $\kappa$-filtered colimits for some regular cardinal $\kappa$.

II.5 Theorem. For each subcategory $\mathscr{A}$ of a locally presentable category of the following are equivalent

(i) $\mathscr{A}$ is small-orthogonal.

(ii) $\mathscr{A}$ is accessibly embedded and closed under limits.

(iii) $\mathscr{A}$ is accessibly embedded and orthogonal.

(iv) $\mathscr{A}$ is reflective and locally presentable.

Proof. The implication (ii) $\rightarrow$ (iv) has been proved in $\left[\mathrm{AR}_{2}\right]$. The statement there is that $\mathscr{A}$ is reflective, but each reflective subcategory of a locally presentable category, closed under $\kappa$-filtered colimits, is clearly locally presentable.

(iv) $\rightarrow$ (i) If $\mathscr{A}$ is locally presentable and reflective in a locally presentable category $\mathscr{K}$, then we can choose $\kappa$ such that $\mathscr{K}$ is locally $\kappa$-presentable and $A$ is closed under $\kappa$-filtered colimits in $\mathscr{K}$. Then $\mathscr{A}=\mathscr{M}^{\perp}$ for the class $\mathscr{M}$ of all reflections of $\kappa$-presentable $\mathscr{K}$-objects in $\mathscr{A}$ (which is essentially small, of course). In fact, it is clear that $\mathscr{A} \subseteq \mathscr{M}^{\perp}$, and to prove $\mathscr{M}^{\perp} \subseteq \mathscr{A}$, express any $K \in \mathscr{M}^{\perp}$ as a $\kappa$-filtered colimit of $\kappa$-presentable objects and use the reflections of those objects.

(i) $\rightarrow$ (iii) Given $\mathscr{M}$ small, choose $\kappa$ larger or equal to the presentation ranks of all domains and codomains of $\mathscr{M}$-morphisms, then $\mathscr{M}^{\perp}$ is closed under $\kappa$ filtered colimits.

(iii) $\rightarrow$ (ii) is obvious. 
II.6 Corollary. Assuming Vopěnka's principle, every reflective (= orthogonal) subcategory of a locally presentable category is small orthogonal.

Proof. By [RTA], every reflective subcategory is closed under $\kappa$-filtered colimits for some $\kappa$. The equation "reflective $=$ orthogonal" has been established in [ART].

II.7 Example [GU, p. 104]. Vopěnka's principle is equivalent to the statement "reflective $\Rightarrow$ small-orthogonal" for locally presentable categories. In fact, assuming the negation of Vopěnka's principle, there exists a quasivariety (= epireflective subcategory) of semigroups which is not small-orthogonal.

II. 8 Remark. As a consequence of II. 6 we obtain an interesting result on torsion theories of modules proved (for the case of abelian groups) by M. Dugas and $\mathrm{G}$. Herden [DuH]. Recall that a class $\mathscr{T}$ of modules is a torsion class provided that it is closed under extensions (i.e., a module $B$ lies in $\mathscr{T}$ whenever it has a submodule $A \in \mathscr{T}$ such that $B / A \in \mathscr{T}$ ) coproducts, and quotients. $\mathscr{T}$ is singly generated provided that it is the least torsion class containing a given module.

II.9 Proposition. Assuming Vopěnka's principle, every torsion class of modules is singly generated.

Proof. Every torsion class $\mathscr{T}$ is a mono-coreflective subcategory. Let $T(A) \rightarrow$ $A$ denote the coreflections. Then the subcategory $\mathscr{F}$ of all modules $F$ with $T(F)=0$ is epireflective. The reflections are $A \rightarrow A / T(A)$. By II.6, II.3, and II.4(b), there is a morphism $m: C \rightarrow C^{\prime}$ with $\mathscr{F}=\{m\}$-Inj. The reflection $r: C \rightarrow C / T(C)$ factors through $m$, since $C / T(C)$ is $m$-injective; thus $\mathscr{F}=$ $\{r\}$-Inj. In other words, a module $B$ lies in $\mathscr{F}$ iff $\operatorname{hom}(T(C), B)=0$. This means that $T(C)$ generates $\mathscr{T}$.

\section{SMALl INJECTIVITY AND WEAK REFLECTIVITY}

III.1 Remark. We will now study small-injectivity classes not only in locally presentable categories but in a wider class of categories. Then we will return to locally presentable categories in $\S \mathrm{IV}$, where small-injectivity classes will be fully characterized.

III.2 Definition. A category is said to be locally ranked provided that it is cocomplete and cowellpowered, and each object $K$ has a rank, i.e., the homfunctor $\operatorname{hom}(K,-)$ preserves $\kappa$-direct colimits of extremal monomorphisms for some regular cardinal $\kappa$.

III.3 Examples. (1) Locally presentable categories are locally ranked.

(2) Top is locally ranked. No nondiscrete topological space $K$ is $\kappa$-generated, i.e., $\operatorname{hom}(K,-)$ does not preserve $\kappa$-direct colimits of monomorphisms (for any $\kappa)$, yet, $\operatorname{hom}(K,-)$ preserves $\kappa$-direct colimits of topological embeddings whenever $\kappa>$ card $K$.

(3) The category Unif of uniform spaces is locally ranked.

(4) The category of compact $T_{2}$-spaces is not locally ranked, although it is a cowellpowered epireflective subcategory of Top.

The category of Urysohn spaces (i.e., spaces in which two distinct points always have disjoint closed neighbourhoods) is not cowellpowered. Thus, this epireflective subcategory of Top is not locally ranked. 
III.4 Proposition. Every small-injectivity class in a locally ranked category is closed under $\kappa$-direct colimits of extremal subobjects (for some regular cardinal $\kappa)$.

Every small-injectivity class in a locally presentable category is accessibly embedded.

Proof. Given a set $\mathscr{M}$ of morphisms, let $\kappa$ be a regular cardinal larger or equal to the ranks of all domains of $\mathscr{M}$-morphisms. Then $\mathscr{M}$-Inj has the stated property.

III.5 Examples. (1) Complete lattices form an injectivity class of posets (I.2), but not a small-injectivity class: they are not accessibly embedded.

(2) Complete Boolean algebras form a small-injectivity class of frames (and a nonsmall injectivity class of Boolean algebras).

(3) Pathwise connected topological spaces form a small-injectivity class of topological spaces.

III.6 Construction of injective weak reflection. We will now show how to construct a weak reflection in $\mathscr{M}$-Inj. It will be clear that if our construction stops, it yields a weak reflection, and in the next theorem we will show that in a wide range of categories the construction always stops. We assume here that $\mathscr{M}$ is small. The argument is every similar to that of $\mathrm{M}$. Kelly [K] who studies reflections in small-orthogonal subcategories.

Let $\mathscr{M}$ be a small collection of morphisms in a cocomplete category $\mathscr{K}$, $\mathscr{M}=\left\{A_{t} \stackrel{m_{t}}{\rightarrow} A_{t}^{\prime} \mid t \in T\right\}$. For each object $K$ of $\mathscr{K}$ we define a chain $w_{i j}: K_{i}^{*} \rightarrow$ $K_{j}^{*} \quad(i, j \in$ Ord,$i \leq j)$ by the following induction.

First step. $K_{0}^{*}=K$.

Isolated step. Given $K_{i}^{*}$, form the canonical map can from $\coprod_{t \in T, f: A_{t} \rightarrow K_{i}^{*}} A_{t}$ into $K_{i}^{*}$ and define $K_{i+1}^{*}$ and $w_{i, i+1}$ by the following pushout

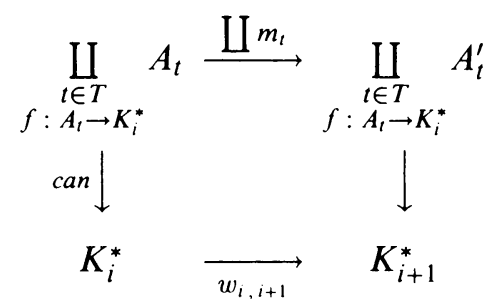

Limit step. $K_{i}^{*}=\operatorname{colim}_{j \leq j^{\prime}<i}\left(K_{j} ; w_{j j^{\prime}}\right)$ with colimit maps $w_{j i}$. We say that the injective weak-reflection construction stops if $w_{i, i+1}$ is a split monomorphism for some $i$. In that case, $w_{0, i}: K \rightarrow K_{i}^{*}$ is a weak reflection of $K$ in $\mathscr{M}$-Inj. In fact

I. $K_{i}^{*}$ is $\mathscr{M}$-injective because can factors through $\amalg m_{t}$, and each morphism $f_{t_{0}}: A_{t_{0}} \rightarrow K_{i}^{*}$ factors through can-thus, $f_{t_{0}}$ factors through $m_{t_{0}}$.

II. If $L$ is $\mathscr{M}$-injective, then for each morphism $g: K \rightarrow L$ we can define a compatible cone $g_{j}: K_{j}^{*} \rightarrow L$ of the above construction as follows: $g_{0}=g$; limit steps are clear; given $g_{j}: L_{j}^{*} \rightarrow L$ then the injectivity of $L$ guarantees that $g_{j}$.can factors through $\amalg m_{t}$ and thus, by the pushout property we get a unique factorization map $g_{j+1}: K_{j+1}^{*} \rightarrow L$. Thus, $g=g_{i} . w_{0 i}$.

III.7 Examples. (1) Consider $m: \phi \rightarrow T$ as in II.2(2), where $T$ is the terminal object. The above construction yields $K_{0}^{*}=K, K_{1}^{*}=K+T, K_{2}^{*}=K+T+$ $T, \ldots, K_{\omega}^{*}=K+T+T+T \cdots$. 


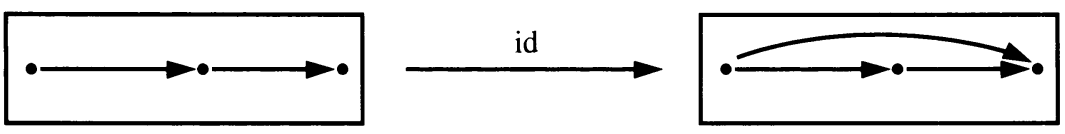

FIGURE 2

The construction stops after $\omega$ steps. However, $K_{1}^{*}$ already is a weak reflection of course.

(2) In Gra consider the morphism $m=$ id pictured in Figure 2. Then $\{m\}$-Inj is the subcategory of transitive relations, and the construction above is the iterative construction of transitive hulls. It clearly stops after $\omega$ steps, and there are objects [e.g.,. $(\omega,\{(n, n+1) \mid n \in \omega\})]$ for which $K_{i}^{*}$ is not a weak reflection for any $i<\omega$.

III.8 Proposition. Let $\mathscr{M}$ be a small collection of morphisms in a locally presentable category, and let $\kappa$ be a regular cardinal larger or equal to the presentation rank of all domains of $\mathscr{M}$-morphisms. Then the injective weak-reflection construction always stops after $\kappa$ steps.

Proof. We will prove that $w_{\kappa, \kappa+1}: K^{*} \rightarrow K_{\kappa+1}^{*}$ is a split monomorphism. Each morphism $f: A_{t} \rightarrow K_{\kappa}^{*}=\operatorname{colim}_{i<\kappa} K_{i}^{*}$ factors as $f=w_{i, \kappa} \cdot \bar{f}$ for some $\bar{f}: A_{t} \rightarrow$ $K_{i}^{*}$ (since $A_{t}$ is $\kappa$-presentable). By the definition of the $i$ th step, there is $\bar{f}^{\prime}: A_{t}^{\prime} \rightarrow K_{i+1}^{*}$ with $\bar{f}^{\prime} \cdot m_{t}=w_{i, i+1} \cdot \bar{f}$. Thus,

$$
f=\left(w_{i+1, \kappa} \cdot w_{i, i+1}\right) \cdot \bar{f}=w_{i+1, \kappa} \cdot \bar{f}^{\prime} \cdot m_{t} .
$$

Let $g: \bigsqcup_{t \in T, f: A_{t} \rightarrow K_{\kappa}^{*}} A_{t}^{\prime} \rightarrow K_{\kappa}^{*}$ be the morphism with components $w_{i+1, \kappa}$. $\bar{f}^{\prime}: A_{t}^{\prime} \rightarrow K_{\kappa^{\prime}}^{*}$ then $g \cdot \amalg m_{t}=$ can: $A_{t} \rightarrow K_{\kappa}^{*}$. By the definition of pushout (in the $(\kappa+1)$ th step) there exists $h: K_{\kappa+1}^{*} \rightarrow K_{\kappa}^{*}$ with $h \cdot w_{\kappa, \kappa+1}=1$.

III.9 Corollary. Let $\mathscr{K}$ be a locally presentable category. Each small-injectivity class is weakly reflective in $\mathscr{K}$, and moreover, there are arbitrarily large regular cardinals $\kappa$ such that each $\kappa$-presentable object of $\mathscr{K}$ has a $\kappa$-presentable weak reflection.

Proof. Let $\mathscr{M}=\left\{A_{t} \stackrel{m_{t}}{\rightarrow} A_{t}^{\prime} \mid t \in T\right\}$ be a small collection of morphisms in $\mathscr{K}$. Consider a cardinal $\alpha$ such that the category $\mathscr{K}$ and all objects $A_{t}$, $t \in T$, have presentation ranks smaller than $\alpha$. For each object $K$ of $\mathscr{K}$ let $|K|=\amalg_{X} \mathscr{K}(X, K)$, where the coproduct ranges over representatives $X$ of the isomorphism classes of $\alpha$-presentable objects of $\mathscr{K}$. There are arbitrarily large regular cardinals $\kappa$ such that $K$ is $\kappa$-presentable iff card $|K|<\kappa$ for any object $K$ of $\mathscr{K}$ (see [GU], or [MP, 2.3.13]). Take such a cardinal $\kappa$ which is greater than $\alpha$ and the presentation ranks of $A_{t}^{\prime}, t \in T$. It is easy to check that $\kappa$ has the desired property.

III.10 Remark. Corollary III.9 can also be derived from [MP, 3.3.1].

The proof of the next result uses methods developed by Max Kelly [K].

III.11 Theorem. Let $\mathscr{K}$ be a locally ranked category. For each small collection $\mathscr{M}$ in $\mathscr{K}$ the injective weak-reflection construction always stops. Thus, $\mathscr{M}-$ Inj is weakly reflective in $\mathscr{K}$.

Proof. (a) We recall an important result of V. Koubek and J. Reiterman [KR]. Let us say that a functor $F$ has rank if there is a regular cardinal $\kappa$ such that 
$F$ preserves $\kappa$-directed colimits of chains of strong monomorphisms. Then for each chain $K_{i}^{*} \quad(i \in$ Ord $)$ in $\mathscr{K}$ there exist ordinals $\alpha(0)<\alpha(1)<\cdots<$ $\alpha(i) \cdots \quad(i<\kappa)$ such that every functor of rank $\kappa$ preserves $\operatorname{colim}_{i<\kappa} K_{\alpha(i)}^{*}$.

(b) Now we apply (a) to the construction of injective weak reflection above. For each $t \in T$ the hom-functor $\operatorname{hom}\left(A_{t},-\right): \mathscr{K} \rightarrow$ Set has rank and its coadjoint preserves colimits. Thus the composite $H_{t}: \mathscr{K} \rightarrow \mathscr{K}$ of the two functors (given by $H_{t} X=\bigsqcup_{f: A_{t} \rightarrow X} A_{t}$ ) has a rank. Thus, the coproduct $\coprod_{t \in T} H_{t}$ also has a rank. Analogously, from $A_{t}^{\prime}$ we get a functor $H_{t}^{\prime}: \mathscr{K} \rightarrow \mathscr{K}$ with a rank and also $\amalg H_{t}^{\prime}$ has a rank. The given morphism $m_{t}: A_{t} \rightarrow A_{t}^{\prime}$ defines an obvious natural transformation $\bar{m}_{t}: H_{t} \rightarrow H_{t}^{\prime}$, and the canonical maps $\bigsqcup_{t \in T} \bigsqcup_{f: A_{t} \rightarrow X} A_{t} \rightarrow X$ define a natural transformation $\overline{c a n}: \bigsqcup_{t \in T} H_{t} \rightarrow$ id . Let us form a (pointwise) pushout of $\overline{c a n}$ and $\bigsqcup_{t \in T} \bar{m}_{t}$

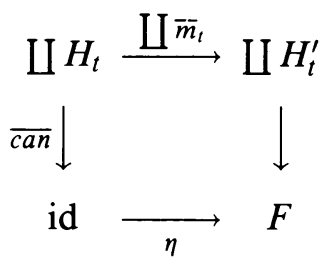

It is clear that a pushout of functors with a rank has a rank. Thus, $F$ is a functor with a rank. By (a), for each object $K$ there exist ordinals $\alpha(i)$ such that $F$ preserves the colimit of $K_{\alpha(i)}^{*}, i<\kappa$. It is easy to check that this implies that the construction of injective weak reflection of $K$ in III.6 stops (after $\alpha(\kappa)$ steps).

III.12 Remark. Analogously, for each locally ranked category $\mathscr{K}, \mathscr{M}^{\perp}$ is reflective in $\mathscr{K}$ whenever $\mathscr{M}$ is a small collection of morphisms. This can be proved using a construction of the reflection which is closely related to III.6 and which was introduced by $M$. Kelly [K, $\S 10]$. In the isolated step one forms the following pushout

$$
\begin{aligned}
\coprod_{t \in T}\left(\coprod_{f: A_{t} \rightarrow K_{i}^{*}} A_{t}+\coprod_{g: A_{t}^{\prime} \rightarrow K_{i}^{*}} A_{t}^{\prime}\right) \rightarrow \coprod_{\substack{t \in T \\
f: A_{t} \rightarrow K_{i}^{*}}} A_{t}^{\prime} \\
\qquad a n \downarrow \\
K_{i}^{*} \underset{w_{i, i+1}}{\downarrow} K_{i+1}^{*}
\end{aligned}
$$

where the upper arrow has the components related to $f$ equal to $m_{t}$, and those related to $g$ equal to $\mathrm{id}_{A_{t}^{\prime}}$. In summary, we get

III.13 Corollary. Let $\mathscr{K}$ be a locally ranked category. Then

(a) each small-orthogonality class in $\mathscr{K}$ is reflective and

(b) each small-injectivity class of $\mathscr{K}$ is weakly reflective.

III.14 Example. The results above do not hold in general for reflective subcategories of locally ranked categories. For example, we have observed above that Frm has a nonreflective, small-orthogonal subcategory of complete Boolean algebras. However, Frm is a reflective subcategory of the category of all posets and all maps preserving all joins and finite meets, and the latter is locally ranked. 
Consequently, it is not in general true that epireflective subcategories inherit the property "small orthogonal $\Rightarrow$ reflective" (any reflective embedding decomposes into two epireflective embeddings ([B]).

\section{A CHARACTERIZATION OF SMALL-INJECTIVITY CLASSES}

IV.1 Remark. Since there are many analogies between the theory of injectivity and orthogonality, one is tempted to expect that the characterization of smallinjective classes in locally presentable categories will be analogous to that of small-orthogonality classes. The latter are just the accessibly embedded classes closed under limits. Unfortunately, an accessibly embedded class closed under products and retracts need not be a small-injectivity class unless we put some set-theoretical restriction. However, an absolute characterization is possible: small-injectivity classes are just those accessibly embedded subcategories which are weakly reflective. In order to prove this result, we will first formulate a simple lemma which, probably, can be found in some form in [GU].

IV.2 Lemma. Let $\mathscr{K}$ be a locally $\kappa$-presentable category. Given a filtered diagram $D: \mathscr{D} \rightarrow \mathscr{K}$, then a compatible cocone $\left(D_{i} \stackrel{c_{i}}{\rightarrow} K\right)_{i \in \mathscr{D}}$ is a colimit of $D$ whenever each $\kappa$-presentable object $L$ has the following properties

(1) every morphism from $L$ to $K$ factors through some $c_{i}$;

(2) given $p, p^{\prime}: L \rightarrow D_{i}$ with $c_{i} \cdot p=c_{i} \cdot p^{\prime}$ then there exists $d: D_{i} \rightarrow$ $D_{j}$ in $\mathscr{D}$ with $d \cdot p=d \cdot p^{\prime}$.

Proof. Since $K$ is a colimit of canonical diagram $\widehat{D}$ w.r.t. the subcategory of all $\kappa$-presentable objects, it is sufficient to show that each compatible cocone $\left(D_{i} \stackrel{c_{i}^{*}}{\rightarrow}\right.$ $\left.K^{*}\right)$ of $D$ "translates" into a compatible cocone of $\widehat{D}$. In fact, each morphism $h: L \rightarrow K$ factors as $h=c_{i} \cdot h^{\prime}$, and we put $h^{*}=c_{i}^{*} \cdot h: L \rightarrow K^{*}$. This is compatible with $\widehat{D}$ since given $f: L \rightarrow L^{\prime}$ in $\widehat{D}$ we have $h \cdot f=c_{j} \cdot(h \cdot f)^{\prime}-$ without loss of generality, $i=j$ (since $D$ is filtered) and $(h \cdot f)^{\prime}=h^{\prime} \cdot f$ (see (2) above), thus, $(h \cdot f)^{*}=h^{*} \cdot f$. Consequently, there exists a unique morphism $k: K \rightarrow K^{*}$ with $h^{*}=k \cdot h$ for each $h: L \rightarrow K$ with $L \quad \kappa$-presentable. Then $c_{i}^{*}=k \cdot c_{i}$ since $D_{i}$ is also a colimit of the canonical diagram, and given $h^{\prime}: L \rightarrow D_{i}$, with $L \quad \kappa$-presentable, then $\left(k \cdot c_{i}\right) \cdot h^{\prime}=k \cdot h=h^{*}=c_{i}^{*} \cdot h^{\prime}$ for $h=c_{i} \cdot h^{\prime}$. The unicity of $k$ w.r.t. $D$ follows from (1) above.

IV.3 Characterization Theorem. In every locally presentable category the smallinjectivity classes are precisely the subcategories which are

(a) weakly reflective and

(b) accessibly embedded.

Proof. The necessity of (a), (b) has been proved in III.9 and III.4. Let $\mathscr{A}$ be a weakly reflective subcategory of a locally presentable category $\mathscr{K}$. If $\mathscr{A}$ is closed under $\kappa$-filtered colimits, we will prove that $\mathscr{A}$ is a small-injectivity class. We may assume that $\mathscr{K}=\operatorname{Set}^{\mathscr{L}}$ for some small category $\mathscr{L}$. (As proved in [GU], each locally presentable category $\mathscr{K}$ can be considered as a reflective subcategory of some $\operatorname{Set}^{\mathscr{L}}$. Thus, we will know that $\mathscr{A}$ is a small-injectivity class of $\operatorname{Set}^{\mathscr{L}}$, and from the reflectivity we will conclude that $\mathscr{A}$ is a smallinjectivity class of $\mathscr{K}$.) In what follows, presentability will be always considered 
in $\mathscr{K}$. Observe that for any regular cardinal $\alpha>\operatorname{card}(\operatorname{Mor}(\mathscr{L}))$ a functor $F: \mathscr{L} \rightarrow$ Set is $\alpha$-presentable iff card $\amalg_{X \in \mathrm{Ob}(\mathscr{L})} F X<\alpha$. Consequently,

(*) every $\alpha^{+}$-presentable object in $\mathscr{K}$, where $\alpha>\operatorname{card}(\operatorname{Mor}(\mathscr{L}))$ is a limit cardinal and $\alpha^{+}$is its successor, is a colimit of an $\alpha$-chain $K_{i}$ $(i<\alpha)$ of objects of presentation ranks smaller than $\alpha$ and with $K_{i}=$ colimit $_{j<i} K_{j}$ for all limit ordinals $i<\alpha$.

I. We will find a regular cardinal $\lambda>\kappa$ such that every $\lambda$-presentable object of $\mathscr{K}$ has a $\lambda$-presentable weak reflection in $\mathscr{A}$. Let

$\delta_{0}=\kappa$

$\delta_{i+1}$ be a regular cardinal such that each $\delta_{i}$-presentable object of $\mathscr{K}$ has a $\delta_{i+1}$-presentable weak reflection in $\mathscr{A}$,

$\delta_{i}=\bigvee_{j<i} \delta_{j}$ for all limit ordinals $i$.

If $\delta_{i+1}=\delta_{i}$ for some $i<\kappa$, we may put $\lambda=\delta_{i}$. Otherwise, $\bar{\delta}=\delta_{\kappa}$ is a limit cardinal and we claim that $\lambda=\bar{\delta}^{+}$has the desired property.

Denote by $\widehat{\mathscr{K}}$ the collection of all $\mathscr{K}$-objects which have a presentation rank smaller than $\bar{\delta}$. Then each $\widehat{\mathscr{K}}$-object has a weak reflection in $\mathscr{A}$ which lies in $\widehat{\mathscr{K}}$. Given a $\lambda$-presentable object $K$, there exists, by $(*)$ above, a $\bar{\delta}$ chain $K_{i} \stackrel{k_{i j}}{\rightarrow} K_{j} \quad(i \leqq j<\bar{\delta})$ of $\widehat{\mathscr{K}}$-objects with colim $K_{i}=\left(K_{i} \stackrel{k_{i}}{\rightarrow} K\right)_{i<\bar{\delta}}$. Let us define a $\bar{\delta}$-chain $K_{i}^{*} \stackrel{k_{i j}^{*}}{\rightarrow} K_{j}^{*}$ in $\mathscr{A} \cap \widehat{\mathscr{K}}$ and a compatible collection $r_{i}: K_{i} \rightarrow K_{i}^{*}$ by the following induction: $r_{0}: K_{0} \rightarrow K_{0}^{*}$ is a weak reflection of $K_{0}$ with $K_{0}^{*} \in \mathscr{A} \cap \widehat{\mathscr{K}}$.

Given $r_{i}: K_{i} \rightarrow K_{i}^{*}$, form the pushout

$$
\begin{array}{cc}
K_{i} \stackrel{k_{i i+1}}{\longrightarrow} & K_{i+1} \\
r_{i} \downarrow & \downarrow_{\bar{r}_{i}} \\
K_{i}^{*} \underset{\bar{k}_{i i+1}}{\longrightarrow} \bar{K}_{i+1} \underset{r_{i+1}^{*}}{\longrightarrow} K_{i+1}^{*}
\end{array}
$$

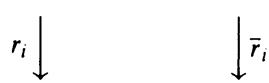

and let $r_{i+1}^{*}: \bar{K}_{i+1} \rightarrow K_{i+1}^{*}$ be a weak reflection of $\bar{K}_{i+1}$ with $K_{i+1}^{*} \in \mathscr{A} \cap \widehat{\mathscr{K}}$. Put $r_{i+1}=r_{i+1}^{*} \cdot \bar{r}_{i}$ and $k_{i, i+1}^{*}=r_{i+1} \cdot \bar{k}_{i, i+1}$.

Given $K_{j}^{*}, j<i$, where $i$ is a limit ordinal, put $\bar{K}_{i}=\operatorname{colim}_{j<i} K_{j}^{*}$ with colimit injections $\bar{k}_{j i}: K_{j}^{*} \rightarrow \bar{K}_{i}$. Let $r_{i}^{*}: \bar{K}_{i} \rightarrow K_{i}^{*}$ be a weak reflection of $\bar{K}_{i}$ with $K_{i}^{*} \in \mathscr{A} \cap \widehat{\mathscr{K}}$. Put $k_{j i}^{*}=r_{i}^{*} \cdot \bar{k}_{j i}$ and $r_{i}=r_{i}^{*} \cdot \operatorname{colim}_{j<i} r_{j}$.

Put $\operatorname{colim}_{i<\bar{\delta}} K_{i}^{*}=\left(K_{i}^{*} \stackrel{k_{i}^{*}}{\rightarrow} K^{*}\right)_{i<\bar{\delta}}$, and observe that $K^{*} \in \mathscr{A}$ since $\bar{\delta}>$ $\kappa$ and $\mathscr{A}$ is closed under $\kappa$-filtered colimits in $\mathscr{K}$. We claim that $r=$ $\operatorname{colim}_{i<\bar{\delta}} r_{i}: K \rightarrow K^{*}$ is a weak reflection of $K$ in $\mathscr{A}$. In fact, given $f: K \rightarrow A$, $A \in \mathscr{A}$, we define a compatible family $f_{i}: K_{i}^{*} \rightarrow A$ with $f \cdot k_{i}=f_{i} \cdot r_{i}$ for all $i<\bar{\delta}$ by the following induction: Choose any $f_{0}: K_{0}^{*} \rightarrow A$ with $f=f_{0} \cdot r_{0}$.

Given $f_{i}$ with $f_{i} \cdot r_{i}=f \cdot k_{i}=\left(f \cdot k_{i+1}\right) \cdot k_{i, i+1}$, there exists a unique $\bar{f}_{i+1}: \bar{K}_{i+1}$ $\rightarrow A$ with $\bar{f}_{i+1} \cdot \bar{k}_{i+1}=f_{i}$ and $\bar{f}_{i+1} \cdot \bar{r}_{i}=f \cdot k_{i+1}$. Choose any $f_{i+1}^{*}: K_{i+1}^{*} \rightarrow A$ with $\bar{f}_{i+1}=f_{i+1}^{*} \cdot r_{i+1}^{*}$.

For each limit ordinal $i$ the choice of $f_{i}$ is clear. The unique morphism $f^{*}: K^{*} \rightarrow A$ with $f^{*} \cdot k_{i}^{*}=f_{i}^{*}(i<\bar{\delta})$ satisfies $f^{*} \cdot r=f \cdot \operatorname{colim}_{i<\bar{\delta}} r_{i}=$ $\operatorname{colim}_{i<\bar{\delta}} f \cdot k_{i}=f$. 
Since each $K_{i}^{*}$ is $\bar{\delta}$-presentable, the colimit $K^{*}=\operatorname{colim}_{i<\bar{\delta}} K_{i}$ is clearly $\lambda$-presentable.

II. To prove that $\mathscr{A}$ is a small-injectivity class, let $K_{j}(j \in J)$ be a small collection representing all $\lambda$-presentable objects of $\mathscr{K}$, and for each $j$ let $r_{j}: K_{j} \rightarrow K_{j}^{*}$ be a weak reflection in $\mathscr{A}$ with $K_{j}^{*} \lambda$-presentable. Then $\mathscr{A}=\left\{r_{j}\right\}_{j \in J}$-Inj. In fact, every $\mathscr{A}$-object is certainly $\left\{r_{j}\right\}$-injective. Conversely, if a $\mathscr{K}$-object $K$ is $\left\{r_{j}\right\}$-injective, we will show that $K \in \mathscr{A}$. Denote by $D$ the canonical diagram of $K$ w.r.t. $\left\{K_{j}\right\}_{j \in J}$, and by $D^{*}$ the canonical diagram of $K$ w.r.t. $\left\{K_{j}^{*}\right\}_{j \in J}$. Since $\lambda \geq \kappa, \mathscr{K}$ is locally $\lambda$-presentable, $D$ is $\lambda$-filtered, and $K=\operatorname{colim} D$. It is sufficient to prove that $D^{*}$ is $\lambda$-filtered and $K=$ colim $D^{*}$. Since $\mathscr{A}$ is closed under $\lambda$-filtered colimits, we then conclude that $K \in \mathscr{A}$.

(a) To prove that $D^{*}$ is $\lambda$-filtered, consider a subdiagram $D_{0}^{*}$ of $D^{*}$ of less than $\lambda$ morphisms. Since $D_{0}^{*} \subseteq D^{*} \subset D$ and $D$ is $\lambda$-filtered, $D$ has an object $f_{0}: K_{j_{0}} \rightarrow K$ which is the codomain of a compatible collection of $D_{0}^{*}$ inside $D$. There exists, by the injectivity of $K$, a morphism $f_{0}^{*}: K_{j_{0}}^{*} \rightarrow K$ with $f_{0}=f_{0}^{*} \cdot r_{j_{0}}$. Then $f_{0}^{*}: K_{j_{0}}^{*} \rightarrow K$ is an object of $D^{*}$ which is the codomain of a compatible collection of $D_{0}^{*}$.

(b) To prove that $\mathscr{K}=\operatorname{colim} D^{*}$, we use Lemma IV.2. (1) Every morphism from a $\lambda$-presentable object $K_{j}^{*}$ into $K$ factors through some $f^{*}: K_{j}^{*} \rightarrow K$ of $D^{*}$ (because $K$ is $r_{j}$-injective) and (2) given $p, p^{\prime}: K_{j} \rightarrow K_{i}$ and given $f: K_{i} \rightarrow K$ with $f \cdot p=f \cdot p^{\prime}$, we have a coequalizer of $p$ and $p^{\prime}$ which, by [GU] has $\lambda$-presentable codomain, say, $c: K_{i} \rightarrow K_{i_{0}}$. For the unique morphism $f_{0}: K_{i_{0}} \rightarrow K$ with $f=f_{0} \cdot c$ there exists $f^{*}: K_{i_{0}}^{*} \rightarrow K$ with $f_{0}=f^{*} \cdot r_{i_{0}}$ (since $K$ is $r_{i_{0}}$-injective). Then $r_{i_{0}} \cdot c:\left(K_{i} \stackrel{f}{\rightarrow} K\right) \rightarrow\left(K_{i_{0}}^{*} \stackrel{f^{*}}{\rightarrow} K\right)$ is a morphism of $D^{*}$ such that $r_{i_{0}} \cdot c \cdot p=r_{i_{0}} \cdot c \cdot p^{\prime}$.

IV.4 Corollary. A subcategory $\mathscr{A}$ of a locally presentable category is a smallinjectivity class iff it is

(i) closed under products,

(ii) accessibly embedded, and

(iii) accessible (see [MP] or [L], i.e., there is a regular cardinal $\kappa$ such that $\mathscr{A}$ has $\kappa$-filtered colimits, and has a small collection of $\kappa$-presentable objects whose closure under $\kappa$-filtered colimits is all of $\mathscr{A}$ ).

In fact, the proof that a small-injectivity class is accessible can be seen from the preceding proof. All weak reflections of $\lambda$-presentable objects in $\mathscr{A}$ which are $\lambda$-presentable have the property that $\mathscr{A}$ is their closure under $\lambda$-filtered colimits. Thus (i)-(iii) are necessary. To prove that (i)-(iii) are sufficient, we first observe that if $\mathscr{H}$ is a set of $\kappa$-presentable objects whose closure under $\kappa$ filtered colimits is $\mathscr{A}$, and if $\mathscr{A}$ is closed under $\kappa$-filtered colimits in a locally $\kappa$-presentable category, then each $\kappa$-presentable object $K$ has a weak reflection in $\mathscr{A}$, viz., the canonical map

$$
r_{K}: K \rightarrow \prod_{H \in \mathscr{H}} \prod_{f: K \rightarrow H} H .
$$

Then $\mathscr{A}=\mathscr{M}$-Inj for the essentially small collection $\mathscr{M}=\left\{r_{k}\right\}:$ the proof is analogous to II in the preceding proof. 
IV.5 Corollary. A category is a small-injectivity class of some locally presentable category iff it is accessible and has products.

Proof. Follows, from Corollary IV.4 because any accessible category admits a limit-preserving accessible full embedding into some $\operatorname{Set}^{\mathscr{L}}$ (for $\mathscr{K} \kappa$-accessible, take all $\kappa$-presentable objects for $\mathscr{L}^{\text {op }}$ and the Yoneda embedding $\mathscr{K} \rightarrow$ $\mathrm{Set}^{\mathscr{L}}$ ).

IV.6 Remark. The above corollary provides an answer to the problem of Makkai [Ma] concerning the characterization of categories of models of regular theories because these categories coincide with small-injectivity classes of locally presentable categories.

IV.7 Corollary. Assuming Vopěnka's principle, in every locally presentable category the small-injectivity classes are precisely the subcategories which are

(a) closed under products, and

(b) accessibly embedded.

In fact, Vopěnka's principle implies that each accessibly embedded subcategory is accessible, see [RTA].

IV.8 Example. Assuming the negation of the semiweak Vopenka's principle, there exists a class of graphs closed in Gra under products, retracts, and filtered colimits, which is not weakly reflective. (Thus, it is not a small-injectivity class, see Theorem III.11.)

In fact, let $L_{i}(i \in$ Ord $)$ be graphs such that $\operatorname{hom}\left(L_{i}, L_{j}\right) \neq \varnothing$ iff $i \geq j$. Let $\mathscr{A}$ be the smallest subcategory of Gra containing each $L_{i}$ and closed under products, retracts, and filtered colimits. For each $A \in \mathscr{A}$ there exist $i \in$ Ord with $\operatorname{hom}\left(L_{i}, A\right) \neq \varnothing$ (because the class of all graphs with that property is closed under products, retracts, and nonempty colimits). It follows that $\mathscr{A}$ does not have a weakly initial object. If $A$ were weakly initial and $\operatorname{hom}\left(L_{i}, A\right) \neq \varnothing$, it would follow that $\operatorname{hom}\left(L_{i}, L_{i+1}\right) \neq \varnothing-$ a contradiction. Thus, $\mathscr{A}$ is not weakly reflective.

IV.9 Open problem. Can an example such as Example IV.8 be found assuming only the negation of Vopènka's principle?

IV.10 Remark. It can be somewhat surprising that Corollaries IV.4 and IV.7 do not include closedness under retracts. The following (probably well-known) lemma explains this. Recall that a category has split idempotents provided that each idempotent $e(: A \rightarrow A, e \cdot e=e)$ factors as $e=f \cdot g$ where $g \cdot f=1$. (For example, each category with equalizers has split idempotents.)

IV.11 Lemma. Let $\mathscr{K}$ be a category with split idempotents, and let $\mathscr{A}$ be a subcategory of $\mathscr{K}$ closed under isomorphisms.

(1) $\mathscr{A}$ is closed under retracts in $\mathscr{K}$ iff $\mathscr{A}$ has split idempotents.

(2) If $\mathscr{A}$ has $\kappa$-filtered colimits, then $\mathscr{A}$ is closed under retracts.

Proof. (1) It is obvious that closedness under retracts implies that $\mathscr{A}$ has split idempotents. Conversely, let $\mathscr{A}$ have split idempotents. Given $A \in \mathscr{A}$ and $B \underset{r}{\stackrel{m}{\rightleftarrows}} A$ in $\mathscr{K}$ with $r \cdot m=1$, we will prove that $B \in \mathscr{A}$. The idempotent 
$e=m \cdot r$ splits in $\mathscr{A}$, i.e., we have $A \underset{f}{\stackrel{g}{\rightleftarrows}} \bar{A} \in \mathscr{A}$ with $m \cdot r=f \cdot g$ and $g \cdot f=1$.

It is easy to compute that the morphisms $g \cdot m: B \rightarrow \bar{A}$ and $r \cdot f: \bar{A} \rightarrow B$ are mutually inverse. Thus, $B$ is isomorphic to $\bar{A} \in \mathscr{A}$, which proves $B \in \mathscr{A}$.

(2) By [MP, 2.2.1], if $\mathscr{A}$ has $\kappa$-filtered colimits, then its idempotents split.

\section{CONE-REFLECTIVE SUBCATEGORIES}

V.1 Definition. A subcategory $\mathscr{A}$ of a category $\mathscr{K}$ is said to be cone reflective provided that for each $\mathscr{K}$-object $K$ there exists a small cone $r_{i}: K \rightarrow A_{i}$ $(i \in I), A_{i} \in \mathscr{A}$, such that every morphism $f: K \rightarrow A, A \in \mathscr{A}$, factors through $r_{i}$ for some $i \in I$. (In other words, the embedding functor fulfils the solution-set condition.)

V.2 Remarks. (1) A cone-reflective subcategory closed under products and retracts [resp. limits] is weakly reflective [resp. reflective]. Thus, CBool is not cone reflective in Frm (Example I.7).

(2) Any accessible and accessibly embedded subcategory of a locally presentable category is cone reflective [MP, 6.1.2].

(3) [C] In the category Tych of Tychonoff spaces all almost compact spaces (i.e., spaces $X$ such that $\beta X$ is the one-point compactification of $X$ ) form a subcategory which is not cone reflective.

V.3 Theorem. Assuming Vopěnka's principle, every subcategory of a locally presentable category is cone reflective.

Proof. The proof is quite analogous to that of Theorem I.9 above. Let $\mathscr{K}$ be locally presentable, and let $\mathscr{A}=\left\{A_{i}\right\}_{i \in \text { Ord }}$ be a full subcategory. Then $\mathscr{A}_{i}=$ $\left\{A_{j}\right\}_{j \leq i}$ is a small, and hence a cone reflective, subcategory of $\mathscr{K}$. Suppose that $\mathscr{A}$ is not cone reflective. Then there is an object $K$ in $\mathscr{K}$ which has no cone reflection in $\mathscr{A}$. Then for each ordinal $i$ there exists an ordinal $d(i)>i$ such that some arrow $r_{i}: K \rightarrow K_{i}^{*}, K_{i}^{*} \in \mathscr{A}_{d(i)}$, factors through none of the arrows with a codomain in $\mathscr{A}_{i}$. Put, in $K \downarrow \mathscr{K}, L_{0}=K \stackrel{r_{0}}{\rightarrow} K_{0}^{*}$, $L_{1}=K \stackrel{r_{d(0)}}{\rightarrow} K_{d(0)}^{*}, \ldots$, and let $E: K \downarrow \mathscr{K} \rightarrow$ Gra be a full embedding. Then for $i<j$ there is no morphism from $E L_{i}$ to $E L_{j}$ in Gra, from which a contradiction with Vopěnka's principle is derived as in Theorem I.9.

V.4 Examples. (1) Assuming the negation of Vopěnka's principle, Gra contains a large discrete subcategory which, of course, fails to be cone reflective. (For example, the empty graph has no cone reflection.) Thus, unlike Theorem I.9, Vopénka's principle is actually equivalent with the statement of Theorem V.3.

(2) Assuming even more, the negation of weak Vopěnka's principle, there are even orthogonal subcategories which are not cone reflective. In fact, Gra then has two reflective subcategories $\mathscr{A}_{1}, \mathscr{A}_{2}$ such that $\mathscr{A}_{1} \cap \mathscr{A}_{2}$ is not reflective, see [ART]. Then $\mathscr{A}_{1} \cap \mathscr{A}_{2}$ is an orthogonality class which, by Remark V.2(2) above, is not cone reflective.

V.5 Definition (see [NS]). For each class $\mathscr{M}$ of small cones in a category $\mathscr{K}$ let $\mathscr{M}$-Inj denote the collection of all $\mathscr{K}$-objects $K$ which are $\mathscr{M}$-injective, i.e., such that for each $\left(m: A \rightarrow A_{i}\right)_{i \in I}$ in $\mathscr{M}$ and each morphism $f: A \rightarrow$ 
$K$ there exists $i \in I$ and a morphism $f^{\prime}: A_{i} \rightarrow K$ with $f=f^{\prime} \cdot m_{i}$. A (small-)cone-injectivity class is a class of objects of the form $\mathscr{M}$-Inj for some (small) class of small cones in $\mathscr{K}$.

V.6 Proposition. Every cone-injectivity class is closed under retracts. Assuming Vopěnka's principle, the converse holds in locally presentable categories: Every class closed under retracts is a cone-injectivity class.

Proof. The first statement is obvious, the latter follows from Theorem V.3. Given a cone-reflective subcategory $\mathscr{A}$ of a locally presentable category $\mathscr{K}$, let $\mathscr{M}$ be the collection of all reflection cones. It is easy to see that the $\mathscr{M}-$ Inj is the closure of $\mathscr{A}$ under retracts.

V.7 Open problem. Is Vopěnka's principle actually needed in Proposition V.6?

The next theorem follows from [GL and L]. The proof is analogous to that of Corollary IV.4.

V.8 Theorem (Guitart-Lair). A subcategory of a locally presentable category is a small-cone-injectivity class iff it is accessible and accessibly embedded.

V.9 Corollary. (1) A category is accessible iff it is a small-cone-injectivity class of a locally presentable category.

(2) Assuming Vopěnka's principle, an accessible category is a small coneinjectivity class in each locally presentable category into which it is fully embedded.

Proof. (1) follows from Theorem V.8 (see Corollary IV.5).

(2) follows from Theorem V.8 and the following fact proved in [RTA]: assuming Vopěnka's principle, each full embedding into a locally presentable category preserves $\kappa$-filtered colimits for some $\kappa$.

V.10 Remark. One can introduce orthogonality with respect to a cone $\left(m_{i}: A \rightarrow A_{i}\right)_{i \in I}$ by requiring that for any $f: A \rightarrow K$ there exist a unique $i \in I$ and a unique morphism $f^{\prime}: A_{i} \rightarrow K$ with $f^{\prime} \cdot m_{i}=f$. Properties of cone-orthogonality classes are analogous to those or orthogonality classes. The role of limits is played by connected limits, and reflective subcategories are replaced by multireflective subcategories in the sense of Diers [D]. For instance; assuming Vopěnka's principle, a subcategory of a locally presentable category is multireflective iff it is closed under connected limits. The details will appear elsewhere.

\section{REFERENCES}

[AK] J. Adámek and V. Koubek, Least fixed point of a functor, J. Comput. System Sci. 19 (1979), 163-177.

[AR $\left.{ }_{1}\right]$ J. Adámek and J. Rosický, Intersections of reflective subcategories, Proc. Amer. Math. Soc. 103 (1988), 710-712.

$\left[\mathrm{AR}_{3}\right]$ - Reflections in locally presentable categories, Arch. Math. (Brno) 25 (1989), 89-94.

[AR $\left.\mathrm{AR}_{3}\right]$, On orthogonal subcategories of locally presentable categories (to appear).

[ART] J. Adámek, J. Rosický, and V. Trnková, Are all limit-closed subcategories of locally presentable categories reflective?, Proc. Categ. Conf. Louvain-la-Neuve, Lecture Notes in Math., vol. 1348, Springer-Verlag, Berlin and New York, 1988, pp. 1-18.

[B] S. Baron, Reflectors as compositions of epi-reflectors, Trans. Amer. Math. Soc. 136 (1969), 499-508. 
[BB] B. Banaschewski and Bruns, Categorical characterization of the MacNeille completion, Arch. Math. (Basel) 18 (1967), 369-377.

[C] W. W. Comfort, Small spaces which "generate" large spaces, Proc. Amer. Math. Soc. 104 (1988), 973-980.

[D] G. Diers, Categories localement multiprésentables, Arch. Math. (Basel) 34 (1980), 344-356.

[Dr] F. Drake, Set theory, North-Holland, Amsterdam, 1974.

[DuH] M. Dugas and G. Herden, Arbitrary torsion classes of abelian groups, Comm. Algebra 11, (1983), 1455-1472.

[Fa] S. Fakir, Objects algébriquement clos et injectifs dans les catégoires localement présentables, Bull. Soc. Math. France 42 (1975).

[F] E. R. Fisher, Vopěnka's principle, universal algebra and category theory, preprint, 1987.

[FK] P. J. Freyd and G. M. Kelly, Categories of continuous functors. I, J. Pure Appl. Algebra 2 (1972), 169-191.

[Fu] L. Fuchs, Infinite abelian groups I, Academic Press, New York, 1970.

[GL] R. Guitart and C. Lair, Calcul syntaxique des moděles et calcul des formules intérnes, Diagrammes 4 (1980).

[GU] P. Gabriel and F. Ulmer, Lokal präsentierbare Kategorien, Lecture Notes in Math., vol. 221, Springer-Verlag, Berlin and New York, 1971.

[H] H. Herrlich, Topological structures and injectivity, Rend. Circ. Mat. di Palermo 12 (1986), 87-93.

[J] T. Jech, Set theory, Academic Press, New York, 1978.

[Jo] P. T. Johnstone, Stone spaces, Cambridge Univ. Press, Cambridge, 1982.

[K] G. M. Kelly, A unified treatment of transfinite constructions for free algebras, free monoids, colimits, associated sheaves, and so on, Bull. Austral. Math. Soc. 22 (1980), 1-84.

[KR.] V. Koubek and J. Reiterman, Categorical constructions of free algebras, colimits, and completions of partial algebras, J. Pure Appl. Algebra 14 (1979), 195-231.

[L] C. Lair, Catégories modélables et catégories esquissables, Diagrammes 6 (1981).

[M] J.-M. Maranda, Injective structures, Trans. Amer. Math. Soc. 110 (1964), 98-135.

[Ma] M. Makkai, A theorem on Bair-exact categories, with an infinitary generalization, Ann. Pure Appl. Logic 47 (1990), 225-268.

[MP] M. Makkai and R. Paré, Accessible categories: The foundations of categorical model theory, Contemp. Math., vol. 104, Amer. Math. Soc., Providence, R.I., 1989.

[NS] I. Németi and I. Sain, Cone-implicational subcategories and some Birkhoff-type theorems, Colloq. Math. Soc. János Bolyai 29 (1982), 535-578.

[RTA] J. Rosický, V. Trnková, and J. Adámek, Unexpected properties of locally presentable categories, Algebra Universalis 27 (1990), 153-170.

[S] J.Schröder, The category of Urysohn spaces is not cowell-powered, Topology Appl. 16 (1983), 237-241.

[SRK] R. M. Solovay, W. N. Reinhardt, and A. Kanamori, Strong axioms of infinity and elementary embeddings, Ann. Math. Logic 13 (1978), 73-116.

Czech Technical University, Technická 2, 16627 Prague 6, Czechoslovakia

E-mail address: adamek@csearn.bitnet

Department of Mathematics, Masaryk University, Janáčkovo nám.2A, 66295, Brno, Czechoslovakia 